\title{
EFFECT OF SMALL CHANGES IN TEMPERATURE ON THE PROPERTIES OF BODIES
}

\author{
By Mayo D. Hersey
}

\section{ABSTRACT}

When it is found necessary to determine the effect of small departures from normal temperature upon some property of a body or system of bodies, such as the stiffness of a steel spring, the vibration frequency of a tuning fork, or the accuracy of an instrument, it is usually done either (a) by a detailed computation or $(b)$ by a direct experiment in which the temperature is actually varied.

After reviewing and illustrating the usual methods of solution a general mathematical treatment of the problem is given, from which two additional methods are derived that can sometimes be usefully applied: (a) A simple calculation, made possible by the theory of dimensions, which does not require any detailed formula for the property in question; and $(b)$ a combined theoretical and experimental solution, in which the experimental factors have been reduced to a minimum and can be determined without varying the temperature of the body itself, provided the thermal properties of the component materials are known.

Finally, it is pointed out that the same treatment can be extended to any other condition analogous to temperature, such as hydrostatic pressure. ${ }^{1}$

\section{CONTENTS}

I. Introduction

II. Examples illustrating the usual methods of solution

1. Stiffness of a flat spring of uniform cross section

2. Torsional stiffness of a wire of circular cross section

3. Vibration frequency of a flat circular diaphragm clamped at the edge -

4. Experimental solution

III. General mathematical theory -

IV. Dimensional theory

1. Proposition İ̃

2. Proposition II

(a) Graphical method

(b) Analytical method -

V. Examples solved by dimensional analysis alone...

1. Period of a pendulum

2. Spring stiffness (pure bending or twisting)

3. Electrical and thermal resistance

VI. Examples solved with the aid of isothermal experiments.....-..-

1. Venturi air-speed indicator

2. Spring stiffness (complex stresses)

3. Vibration frequency of a loaded spring (elastic pendulum) --

4. Coefficient of friction of a lubricated journal bearing-.---

VII. Extension to determine the effect of physical conditions other than temperature

VIII. Further questions for investigation

Page

138

138

139

139

140

141

141

143

144

145

145

146

146

146

147

148

149

149

151

151

152

154

155

155

1 Recent investigations in which it was found necessary to compute the effect of changes in temperature and in pressure on the properties of bodies have been published by E. A. Harrington, J. Opt. Soc. Am., 18, pp. 89 to 95; 1929; and by P. W, Bridgman, Proc. Am. Acad. Arts. Sci., 63, pp. 401 to 420; 1929. 


\section{INTRODUCTION}

In physical and engineering investigations involving the properties of bodies (as distinguished from substances or materials) it is often necessary to know at least approximately what will be the effect of small departures from the normal working temperature. If the magnitude of the property in question is represented by $P$, the effect of any small change in temperature, $\Delta t$, can obviously be obtained from the relation

$$
\frac{\Delta P}{P}=\left(\frac{1}{P} \frac{d P}{d t}\right) \Delta t
$$

provided the value of the temperature coefficient $(1 / P)(d P / d t)$ is known.

The quantity $P$ may represent, for example, some familiar physical property of a single homogeneous body or some performance characteristic of an instrument or machine. The magnitude of the temperature coefficient $(1 / P)(d P / d t)$ must obviously depend upon various different factors, including the physical properties of the component materials.

The general problem considered in this paper is that of determining the value of the temperature coefficient $(1 / P)(d P / d t)$ or, in other words, the value of the fractional or relative rate of change of the property in question with respect to temperature.

The usual practice is to compute the temperature coefficient from a detailed formula for the property $P$ if such a formula can be written, otherwise to have recourse to a direct experiment in which the values of $P$ are observed at different temperatures. In this paper two additional methods of solution are described after first reviewing and illustrating the more usual methods.

\section{EXAMPLES ILLUSTRATING THE USUAL METHODS OF SOLUTION}

The usual methods of solution, particularly the computation method, can readily be illustrated by three simple examples taken from the field of elasticity, the first involving pure bending, the second pure twisting, and the third involving complex stresses.

These examples will at the same time serve to illustrate the use of logarithmic differentiation and the use of the dot above any quantity to denote the fractional or relative rate of change of that quantity with respect to temperature $(1,2) \cdot{ }^{23}$ For convenience both short cuts will be employed throughout the paper. This present use of the dot to indicate logarithmic differentiation with respect to temperature need not be confused with its use to represent ordinary differentiation with respect to time in mechanics. The logarithmic derivative of any quantity $Q$ with respect to $t$ is defined by the expression $d \log Q / d t$ and therefore is equal to $(1 / Q)(d Q / d t)$ and will be denoted simply by $\dot{Q}$.

\footnotetext{
2 Figures in parentheses here and throughout the text refer to the numbers under "References" at the end of this paper.

${ }^{3}$ Previously used with advantage in papers on The Theory of the Stiffness of Elastic Systems, and Vibration Frequencies of Elastic Systems $(1,2)$.
} 


\section{STIFFNESS OF A FLAT SPRING OF UNIFORM CROSS SECTION}

Let the length, breadth, and depth (or thickness) of the spring be denoted by $L, B$, and $D$, respectively. Consider the spring fixed at one end like a cantilever beam and loaded at the other end by the application of a force $F$. Let $Y$ denote the deflection of the spring at the extreme end due to the force $F$. The spring stiffness $S$ may be defined by the expression $F / Y$ and corresponds, in this example, to the general property $P$, whose temperature coefficient is desired.

Under the foregoing conditions the usual formula for the deflection of a cantilever beam leads to the relation

$$
S=\frac{B E D^{3}}{4 L^{3}}
$$

in which $E$ is Young's modulus of elasticity. Differentiating logarithmically to obtain the effect of temperature change,

$$
\frac{1}{S} \frac{d S}{d t}=\frac{1}{B} \frac{d B}{d t}+\frac{1}{E} \frac{d E}{d t}+\frac{3}{D} \frac{d D}{d t}-\frac{3}{L} \frac{d L}{d t}
$$

or more simply

$$
\dot{S}=\dot{B}+\dot{E}+3 \dot{D}-3 \dot{L}
$$

If the material of the spring can be treated as homogeneous and isotropic, so that the body expands uniformly in all directions without distortion, $\dot{B}=\dot{D}=\dot{L}$ and equation (4) become

$$
\dot{S}=\dot{L}+\dot{E}
$$

This result amounts to the statement that the temperature coefficient of the stiffness of the spring is equal to the linear thermal expansivity of the material plus the temperature coefficient of Young's modulus. For the usual materials, $\dot{L}$ is intrinsically positive while $\dot{E}$ is intrinsically negative.

\section{TORSIONAL STIFFNESS OF A WIRE OF CIRCULAR CROSS SECTION}

Let $M$ denote the toique or moment needed for maintaining an angular deflection $\theta$ at the free end of a vertical wire of length $L$ and diameter $D$. From the theory of elasticity, if the material is isotropic and homogeneous,

$$
M=\frac{\pi D^{4} \mu}{32 L} \cdot \theta
$$

in which $\mu$ denotes the shear modulus of elasticity. Defining the torsional stiffness $T$ by the ratio $M / \theta$, that is, torque per unit twist, equation (6) gives

$$
T=\frac{\pi D^{4} \mu}{32 L}
$$

Differentiating logarithmically with respect to the temperature and making use of the fact that $\dot{D}=\dot{L}$, we obtain

$$
\dot{T}=3 \dot{L}+\dot{\mu}
$$


again a linear function of the thermal expansivity and the temperature coefficient of one of the elastic moduli, this time the temperature coefficient of the shear modulus.

\section{VIBRATION FREQUENCY OF A FLAT CIRCULAR DIAPHRAGM, CLAMPED AT THE EDGE}

Let $n$ denote the frequency of the slowest vibration of a diaphragm of thickness $L$ and diameter $D$, composed of an isotropic, homogeneous, perfectly elastic material of density $\rho$, Young's modulus $E$ and Poisson's ratio $\sigma$. According to Rayleigh (3) ${ }^{4}$ after converting into the above notation and working out an approximate value of the numerical factor

$$
n=\frac{1.88}{\sqrt{1-\sigma^{2}}}\left(\frac{L}{D^{2}}\right) \sqrt{\frac{E}{\rho}}
$$

Differentiating as before, and making use of the identity $d \sigma / d t=\sigma \dot{\sigma}$, we find that

$$
\dot{n}=\left(\frac{\sigma^{2}}{1-\sigma^{2}}\right) \dot{\sigma}+\dot{L}-2 \dot{D}+\frac{\dot{E}}{2}-\frac{\dot{\rho}}{2}
$$

Since, however, $\dot{D}=\dot{L}$ and $\dot{\rho}=-3 \dot{L}$ for isotropic materials, equation (10) can be written

$$
\dot{n}=\left(\frac{\sigma^{2}}{1-\sigma^{2}}\right) \dot{\sigma}+\frac{\dot{L}}{2}+\frac{\dot{E}}{2}
$$

This gives the result as a linear function of three of the temperature coefficients of the material, one of which, the temperature coefficient of Poisson's ratio, can be replaced by other data that are more readily a vailable.

Thus from the theory of elasticity, for the type of material under consideration, the three elastic constants $E$, $\mu$, and $\sigma$ are connected by a definite relation,

$$
\sigma=\frac{1}{2} \frac{E}{\mu}-1
$$

from which it is evident that it should be possible to express $\dot{\sigma}$ in terms of $\dot{E}$ and $\dot{\mu}$. Differentiating, we obtain

$$
\dot{\sigma}=\left(\frac{1+\sigma}{\sigma}\right)(\dot{E}-\dot{\mu})
$$

Accordingly equation (11) reduces to

$$
\dot{n}=\frac{\dot{L}}{2}+\frac{1}{2}\left(\frac{1+\sigma}{1-\sigma}\right) \dot{E}-\left(\frac{\sigma}{1-\sigma}\right) \dot{\mu}
$$

a linear function of the thermal expansivity, temperature cofficient of Young's modulus, and temperature coefficient of the shear modulus.

\footnotetext{
- An alternative formula involving total mass in place of density is given in (2), footnote 3, p. 444.
} 
Taking Poisson's ratio $=3 / 10$ as a rough approximation gives for equation (14)

$$
\dot{n}=\frac{\dot{L}}{2}+\frac{13}{14} \dot{E}-\frac{3}{7} \dot{\mu}
$$

from which the approximate magnitude of $\dot{n}$ can readily be computed for various materials upon consulting tables of published experimental data for the appropriate values of $\dot{L}, \dot{E}$, and $\dot{\mu}$.

\section{EXPERIMENTAL SOLUTION}

To determine the temperature coefficient of any property $P$ when the detailed formula for this property as exemplified by equations (2), (7), or (9) is not available, the most obvious and usual procedure is to set up the apparatus in question and make tests in which the temperature is actually varied. The temperature must be appropriately controlled, allowing time for thermal equilibrium to be reached. Preferably observations of $P$ should be taken both above and below the normal working temperature at which the value of $\dot{P}$ is required. A sufficient range of temperature should be covered so that in plotting $P$ against $t$ the slope can be determined graphically with the requisite precision. Dividing the slope (in proper units) at any given temperature by the value of $P$ at that temperature gives the corresponding value of the temperature coefficient $\dot{P}$.

The observations may be extended, if desired, over a sufficient range of temperature to permit plotting $\dot{P}$ as a function of temperature. This type of experiment, however, can not do more than provide discrete numerical values of $\dot{P}$ applicable to the identical apparatus under test. It does not throw any light on the functional relationship between $\dot{P}$ and the temperature coefficients of the component materials, $\dot{L}, \dot{E}, \dot{\mu}$, etc., as illustrated by equations (5), (8), or (14). Also it requires a specialized laboratory technique in order to work with actual changes of temperature.

\section{GENERAL MATHEMATICAL THEORY}

From a consideration of the nature of the variables entering the problem it will readily become apparent why the solution in every case takes the form of a linear function of the thermal expansivity and other thermal properties ${ }^{5}$ of the component materials.

The character of a body or system of bodies depends evidently upon its size and shape and upon the properties of the component materials. If the system is homogeneous and isotropic, it can be defined by some linear magnitude $L$, together with as many length ratios $r_{1} \ldots r_{l}$ as may be needed to fix the geometrical shape of the system, and the appropriate properties of materials, $p_{1} \ldots p_{m}$.

When the system is not homogeneous and isotropic, the distribution of the values of each one of the $p$ 's must be indicated. This can most readily be done by selecting one point, and, if necessary, one plane and one stated direction at that point, where the absolute magnitude of the property will be given, and then expressing the distribution of the property by ratios of other values to this one.

"For brevity, the term "thermal properties" will be used collectively to denote the temperature coefficients of the properties of the materials, together with their thermal expansivities. 
When two bodies of unequal absolute size possess the same distribution of length ratios, they are said to be geometrically similar or to have the same geometrical shape. By analogy, two bodies or systems of bodies may be said to have the same generalized shape when they possess the same relative distribution of properties (1). ${ }^{6}$

A system which is not homogeneous and isotropic can, therefore, be completely identified or described by giving its linear magnitude $L$ and the magnitudes of the properties $p_{1} \cdots p_{m}$, together with all ratios of like quantities, $r_{1} \ldots r_{l}$, including both ratios of linear magnitudes and ratios of properties of materials, that may be needed to specify the generalized shape.

The property $P$ may depend not only on the intrinsic character of the system as defined by $L, r_{1} \cdots r_{l}$, and $p_{1} \cdots p_{m}$, which may be termed internal variables, but also on a number of other quantities, $q_{1} \ldots q_{n}$, which might be termed the external variables, and which, in general, are independent of temperature. Thus the entire list of independent variables governing the magnitude of $P$ can be divided into the four groups or classes mentioned above and we may write

$$
P=f\left(L, r_{1} \ldots r_{l}, p_{1} \ldots p_{m}, q_{1} \ldots q_{n}\right)
$$

where the form of the function $f$ may or may not be known.

As a step toward investigating the effect of temperature on the property $P$ in the light of equation (16) let us consider any system of $N$ physical quantities $Q_{o}, Q_{1}, \cdots Q_{N-1}$ which are definitely related, so that

$$
Q_{o}=f\left(Q_{1}, \cdots Q_{N-1}\right)
$$

By ordinary differentiation

$$
d Q_{o}=\left(\frac{\partial Q_{o}}{\partial Q_{1}}\right) d Q_{1}+\cdots
$$

Hence by logarithmic differentiation

or

$$
\frac{d Q_{0}}{Q_{o}}=\left(\frac{\partial Q_{o} / Q_{0}}{\partial Q_{1} / Q_{1}}\right) \frac{d Q_{1}}{Q_{1}}+\cdots=\left(\frac{\partial \log Q_{0}}{\partial \log Q_{1}}\right) \frac{d Q_{1}}{Q_{1}}+\cdots
$$

$$
\dot{Q}_{o}=\left(\frac{\partial \log Q_{o}}{\partial \log Q_{1}}\right) \dot{Q}_{1}+\cdots
$$

The same computation can now be applied to equation (16), taking $P$ as the equivalent of $Q_{o}$, and the quantities $L, r_{1} \cdots r_{l}, p_{1} \cdots p_{m}$, $q_{1} \cdots q_{n}$ as equivalent to $Q_{1} \cdots Q_{N-1}$. Since in general $q_{1} \cdots q_{n}$ are wholly unaffected by temperature, we obtain

$$
\dot{P}=\left(\frac{\partial \log P}{\partial \log L}\right) \dot{L}+\left(\frac{\partial \log P}{\partial \log r_{1}}\right) \dot{r}_{1}+\cdots+\left(\frac{\partial \log P}{\partial \log p_{1}}\right) \dot{p}_{1}+\ldots+\left(\frac{\partial \log P}{\partial \log p_{m}}\right) \dot{p}_{m}
$$

From equation (21) it is clear that $\dot{P}$ must be a linear function of the thermal properties $\dot{L}, \dot{r}_{1} \cdots \dot{r}_{l}, \dot{p}_{1} \cdots \dot{p}_{m}$ in all cases, regardless of the form of the function $f$.

' See p. 571 for rolled sheet metal as an illustration of bodies having the same generalized shape, and for further discussion of this concept. Two pendulums of different sizes having variable densities similarly distributed constitute another such example. 
When the generalized shape can be treated as sensibly constant, $\dot{r}_{1} \ldots \dot{r}_{l}$ may be set equal to zero, and then equation (21) reduces to

$$
\dot{P}=\left(\frac{\partial \log P}{\partial \log L}\right) \dot{L}+\left(\frac{\partial \log P}{\partial \log p_{1}}\right) \dot{p}_{1}+\cdots\left(\frac{\partial \log P}{\partial \log p_{m}}\right) \dot{p}_{m}
$$

or more simply

$$
\dot{P}=C_{o} \dot{L}+C_{1} \dot{p}_{1}+\cdots+C_{m} \dot{p}_{m}
$$

in which $C_{o}, C_{1}, \cdots C_{m}$ denote the dimensionless, isothermal coefficients $(\partial \log P / \partial \log L),\left(\partial \log P / \partial \log p_{1}\right)$, etc.

The restriction of equation (23) to a constant generalized shape is not a prohibitive one because (a) for homogeneous and isotropic bodies the generalized shape is the same as the geometrical shape and is actually constant; (b) for many bodies that are not homogeneous and isotropic the variation of $r_{1} \cdots r_{l}$ with temperature is a second-order effect which can be neglected; and (c) when not negligible, as, for example, in considering the efficiency of a machine built of cast iron having its bearings fitted with brass bushings, the effect of temperature on the ratios $r_{1} \cdots r_{l}$ can sometimes be calculated in detail by conventional methods and superposed on the solution obtained for a system of constant generalized shape.

From equation (23) it is evident that a complete experimental solution can be obtained without varying the temperature, if appropriate experiments are made for evaluating the isothermal coefficients $C_{o}, C_{1}, \cdots C_{m}$, and provided the thermal properties of the component materials are already known. These are usually available in published tables to a sufficient degree of approximation for the purpose in hand; if not, they can be determined by testing the component materials as such, without reference to the particular form of the bodies in question.

In actual practice the procedure suggested by equation (23) can be further simplified, as will be shown by means of the theory of dimensions.

\section{DIMENSIONAL THEORY}

A number of very interesting conclusions can be deduced from equation (17) if it is understood in what follows that this equation expresses a qualitatively complete relation. If it is incompletethat is, if any essential quantity has been overlooked in making up the list of independent variables $Q_{1} \cdots Q_{N-1}$ on which the value of $Q_{0}$ depends-we should not be warranted in applying the following analysis. If, however, the equation is overcomplete - that is, contains one or more quantities that are superfluous-no harm will be done and the extra variables introduced will automatically drop out again later.

For convenience equation (17) will further be restricted to systems of constant generalized shape or to different systems of the same generalized shape, so that the ratios $r_{1} \cdots r_{l}$ need not be explicitly mentioned, and therefore the variables $Q_{o}, Q_{1} \cdots Q_{N-1}$ represent $N$ different kinds of physical quantities.

It is also understood that the form of the function $f$ is entirely unknown, for if it were already known we should have nothing to gain by further inquiry and could proceed at once to the usual solution by differentiation illustrated by three examples earlier in the paper.

$84789^{\circ}-29-10$ 
It follows from Buckingham's $\Pi$-theorem $(4,5)$ that equation (17) must be reducible to the form (7)

$$
\Pi_{o}=\phi\left(\Pi_{1}, \Pi_{2}, \cdots \Pi_{j}\right)
$$

in which the function $\phi$ is unknown, but in which the ח's are known and represent all of the independent dimensionless products that can be formed from the $N$ physical quantities involved, and in which $j=N-k-1$, where $k$ is the number of fundamental units (6) needed for measuring the $N$ quantities.

In equation $(24) \Pi_{o}$ represents the dimensionless product containing $Q_{o}$, together with as many of the quantities $Q_{1} \cdots Q_{k}$ as may be found necessary to cancel out the dimensions of $Q_{o}$; that is,

$$
\Pi_{o}=\left(Q_{1}{ }^{a_{0}} Q_{2}{ }^{\beta_{o}} \ldots Q_{k}^{\kappa_{o}}\right) Q_{o}
$$

in which the exponents $\alpha_{o}, \beta_{o}, \cdots \kappa_{o}$ are pure numbers, some of which may be zero in any concrete example. In like manner we can write for any one of the products $\Pi_{1} \cdots \Pi_{j}$ serving as arguments of the unknown function $\phi$,

$$
\Pi=\left(Q_{1}{ }^{a} Q_{2}{ }^{\beta} \cdots Q_{k}{ }^{k}\right) Q
$$

Substituting from equation (25) into equation (24) and solving for $Q_{0}$ gives finally

$$
Q_{o}=Q_{1}^{-a_{\circ}} Q_{2}{ }^{-\beta_{o}} \cdots Q_{k}^{-\kappa_{o}} \phi\left(\Pi_{1}, \mathrm{II}_{2}, \cdots \Pi_{j}\right)
$$

Upon identifying the general quantities $Q_{o}, Q_{1}, \cdots Q_{N-1}$ of equation (27) with the classified quantities $P, L, p_{1} \cdots p_{m}$ and $q_{1} \cdots q_{n}$ of equation (16), and omitting $r_{1} \cdots r_{l}$ by virtue of the restriction to systems of the same generalized shape, two propositions of practical interest can be deduced, as shown below.

\section{PROPOSITION I}

When $N=k+1$, that is, when the number of different kinds of physical quantity entering the problem exceeds the number of fundamental units by one, as frequently occurs in practice, the unknown function $\phi$ reduces to a pure number, and is therefore constant.

If when $N$ is greater than $k+1$, the arguments $\Pi_{1} \cdots \Pi_{j}$ entering the unknown function can be constructed solely from the external variables $q_{1} \ldots q_{n}$, which are independent of temperature, $\phi$ can be treated as a constant with respect to temperature.

Again, if one or more of the arguments entering the unknown function does contain the linear magnitude $L$ or properties $p_{1} \cdots p_{m}$ it may, in some applications, be found upon inspection that these quantities tend to balance one another in such a way that the net effect of temperature on the product in question can be neglected with a satisfactory degree of approximation. This possibility is especially noteworthy in problems where it can be shown that the product or products affected have only a slight influence on the value of $\phi$.

In any case where $\phi$ can be treated as a constant with respect to temperature it will be denoted by $\phi_{o}$ and equation (27) becomes

$$
Q_{o}=\phi_{o} \cdot Q_{1}^{-\alpha_{o}} Q_{2}^{-\beta_{o}} \cdots Q_{k}^{-\kappa_{o}}
$$


Changing over to the classified notation we obtain for $P$ the continued product

$$
P=\text { const. } L^{x_{0}} p_{1}^{x_{1}} \cdots p_{m}^{x_{m}} q_{1}^{y_{1}} \cdots q_{n}^{y_{n}}
$$

in which $x_{0} \cdots x_{m}$ denote the numerical exponents of the internal variables and $y_{1} \cdots y_{n}$ those of the external variables. The number of factors, $m+n+1$, can not exceed the number of fundamental units, $k$.

Differentiating equation (29) logarithmically and making use of the fact that $q_{1} \cdots q_{n}$ are uninfluenced by changes of temperature, we find that

$$
\dot{P}=x_{o} \dot{L}+x_{1} \dot{p}_{1}+\cdots+x_{m} \dot{p}_{m}
$$

Comparing equation (30) with equation (23) it is evident that $C_{o}=x_{o}$, $C_{1}=x_{1}, \cdots C_{m}=x_{m}$.

Thus whenever the unknown function $\phi$ can be treated as a constant with respect to temperature, the coefficients entering equation (23) may be found by inspection without the aid of experiment.

\section{PROPOSITION II}

In the general case where $\phi$ must be treated as a variable the coefficients of $\dot{L}, \dot{p}_{1} \cdots \dot{p}_{m}$ entering the formula for $\dot{P}$, equation (23), can not be determined solely by computation. Two methods are available, however, mathematically identical in substance, one graphical and the other analytical, by which the experimental work can be reduced to a minimum.

(a) Graphical Method.-Let $K$ denote the slope, at any point, of an isothermal curve obtained by plotting $\log \Pi_{o}$ as ordinate against $\log \Pi$ as abscissa, where $\Pi_{o}$ and $\Pi$ are defined by equations (25) and (26). If the function $\phi$ has only one argument, $\Pi$, we have

$$
\Pi_{o}=\phi(\Pi)
$$

so that equation (27) can be written

$$
Q_{o}=Q_{1}^{-a_{0}} Q_{2}-\beta_{0} \cdots \phi(\Pi)
$$

Differentiating to obtain the temperature coefficient of the property $P$ which is represented by $Q_{o}$ in the foregoing notation, we find

$$
\dot{Q}_{o}=-\alpha_{o} \dot{Q}_{1}-\beta_{o} \dot{Q}_{2}-\ldots+\left(\frac{d \log \phi}{d \log \Pi}\right) \dot{\Pi}
$$

But from equation (31) $\phi=\Pi_{o}$, so that $d \log \phi / d \log \Pi$ is equal to the slope $K$; and from equation $(26), \dot{\Pi}=\dot{Q}+\alpha Q_{1}+\beta \dot{Q}_{2}+\ldots$; therefore

$$
\dot{Q}_{o}=K \dot{Q}+\left(\alpha K-\alpha_{o}\right) \dot{Q}_{1}+\left(\beta K-\beta_{o}\right) \dot{Q}_{2}+\ldots
$$

The same result can be obtained by putting equation (31) in the form $\Pi_{o} \propto \Pi^{K}$, substituting from equations (25) and (26), differentiating and solving for $\dot{Q}_{o}$, treating $K$ as a constant in the immediate vicinity of any given point on the curve. 
In the rare event when we are obliged to deal with more than one argument $\Pi$, this result will be replaced by a summation of terms on the right-hand side, in which $K$ denotes in succession each one of the partial derivatives.

The simplification accomplished by equation (34), as compared to equation (23), consists in three facts:

(1) The experiments can be performed on geometrically similar models, $i^{f}$ preferred, instead of on the original system of bodies. ${ }^{7}$

(2) Only one quantity need be varied in conducting the isothermal observations; therefore if more than one quantity can conveniently be varied, an absolute check is available.

(3) The quantity selected for experimental variation need not necessarily be the linear dimension or one of the properties of the component materials, since, if preferred, any one of the external variables entering the product will serve equally well.

(b) Analytical Method.-If $Q_{1}$ and $Q_{2}$ are any two quantities that occur in either or both of the dimensionless products $\Pi_{o}$ and $I$, the remaining products being held constant for the time, it follows from the relation connecting the derivatives of physical quantities (7) that

$$
\frac{\partial \log Q_{o}}{\partial \log Q_{1}}=\left(\frac{\alpha}{\beta} \beta_{o}-\alpha_{o}\right)+\left(\frac{\alpha}{\beta}\right) \frac{\partial \log Q_{o}}{\partial \log Q_{2}}
$$

In applying this formula to equation (23), $Q_{o}$ is taken as before to represent $P$, while $Q_{1}$ represents any one of the internal variables $L$, $p_{1} \ldots p_{m}$, and $Q_{2}$ may represent either an internal or an external variable; that is, it may be allowed to represent any one of the quantities $L, p_{1} \ldots p_{m}, q_{1} \ldots q_{n}$, subject to the restrictions discussed in the reference cited $(7)$.

In practice the values of $\alpha_{o}, \beta_{o}, \alpha$, and $\beta$ are found by inspection upon comparing the actual dimensional expression for the property $P$ with the symbolic expression given by equations (25) and (26), while the value of the partial derivative $\partial \log Q_{o} / \partial \log Q_{2}$ is obtained from the isothermal observations.

The use of equation (35) in conjunction with equation (23) has the same ultimate advantages as the use of the graphical formula (34) and should give the same result; therefore, in any particular application one method can be employed as a check on the other.

\section{EXAMPLES SOLVED BY DIMENSIONAL ANALYSIS ALONE}

\section{PERIOD OF A PENDULUM}

Let $T$ denote the period of any rigid compound pendulum of heterogeneous construction, whose generalized shape might be specified by various length ratios and density ratios $r_{1} \ldots r_{l}$. Then if $g$ denotes the acceleration of gravity, while $L$ represents any chosen linear dimension,

$$
T=f\left(L, g, r_{1} \ldots r_{\imath}\right)
$$

7 The requisite conditions have been fully discussed by Buckingham (5) and consist essentially in securing the same value of $I I$ for both model and original. 
in which the function $f$ will be considered unknown. Applying the $\Pi$-theorem $;^{8}$ that is, rewriting equation (36) in the standard II-theorem form which involves dimensionless variables only and then solving for $T$, we obtain

$$
T=\sqrt{\frac{L}{g}} \phi\left(r_{1} \cdots r_{l}\right)
$$

To a high degree of approximation for small changes in temperature the generalized shape may be treated as a constant with respect to temperature, and since $g$, being an external variable, is also independent of temperature, we find simply

$$
\dot{T}=\frac{1}{2} \dot{L}
$$

This example, therefore, is an illustration of Proposition I and might have been solved by direct substitution in equation (30), taking $P=T, L=L$, and $x_{o}=1 / 2$.

\section{SPRING STIFFNESS (PURE BENDING OR TWISTING)}

For springs of irregular shape, such that no detailed formula equivalent to equation (2) is available, we can write, in the case of pure bending,

$$
S=f\left(L, E, r_{1} \cdots r_{l}\right)
$$

in which $f$ is an unknown function, while $L$ denotes a linear dimension, and $E$ denotes Young's modulus either at some arbitrary point in the material or the mean value throughout. The length ratios and ratios of Young's modulus, $r_{1} \cdots r_{l}$, taken together, serve to define the generalized shape of the spring. By dimensional analysis, as before, equation (39) becomes

$$
S=\operatorname{LE\phi }\left(r_{1} \cdots r_{l}\right)
$$

in which $\phi$ is an unknown function. Treating the generalized shape as a constant with respect to temperature, which permits a moderate but not an excessive departure from the homogeneous, isotropic condition due to rolling, stamping, or heat treatment, we have

$$
\dot{S}=\dot{L}+\dot{E}
$$

This result also might have been obtained from equation (30) by substituting $P=S, L=L, p_{1}=E, x_{o}=1$, and $x_{1}=1$.

If instead of pure bending we have a spring deformed by pure twisting or shearing, the solution is the same as before, except that Young's modulus $E$ will be replaced by the shear modulus $\mu$ in equations (39) to (41).

The solution for the torsional stiffness of a vertical suspension (wire, ribbon, or fiber) of any cross-sectional form can be shown by ${ }^{8}$ See references (4) and (5). The II-theorem can be applied by inspection after a little practice if the
dimensions of the various quantities are îrst written down for ready comparison. Further information on the method of dimensions will be found in references (8), (9), (10), and (11), 
dimensional analysis to be the same as was given for the circular wire in equation (8).

The solution for complex stresses, that is, where bending and shearing deformations occur simultaneously, can not be obtained from dimensional analysis alone without experimental data and will, therefore, be treated later as an application of Proposition II.

\section{ELECTRICAL AND THERMAL RESISTANCE}

Let $R$ denote the resistance of any electrically conducting body of irregular shape for which the detailed formula is not available; then if $L$, as before, represents a linear dimension of this conductor, while the resistivity of the material is denoted by $\rho$,

$$
R=f\left(L, \rho, r_{1} \cdots r_{l}\right)
$$

For conductors of constant shape we obtain from equation (42) by the method of dimensions

$$
R=\frac{\rho}{L} \cdot \phi_{o}
$$

where $\phi_{o}$ is a constant, and, therefore

$$
\dot{R}=\dot{\rho}-\dot{L}
$$

Thus the temperature coefficient of resistance of the body is equal to the temperature coefficient of the volumetric resistivity of the material minus its linear thermal expansivity, a result which can be checked by the usual method of solution in the particular case of any body of simple geometrical form. Equation (44) will be recognized as a consequence of equation (30) if we put $P=R, L=L, p_{1}=\rho$, $x_{o}=-1$, and $x_{1}=1$.

In the analogous problem of heat conduction the same notation can be employed, $R$ being interpreted as the thermal resistance of the body (temperature drop per unit flow of heat) and $\rho$ as the thermal resistivity of the material (reciprocal of thermal conductivity). In the thermal problem, however, the temperature of the body can not be assumed uniform, since the existence of a finite temperature drop, $\Delta t$, is essential to the process of heat conduction.

The material of the thermally conducting body is therefore, in general, nonhomogeneous and departs more and more from homogeneity as the temperature drop increases. Mathematically this signifies that the length ratios and resistivity ratios $r_{1} \ldots r_{l}$ corresponding to any given reference temperature $t$ (for example, the mean temperature of the body or the temperature at the center of the cooler surface) must be recognized as functions of $\Delta t$.

If the length ratios are functions of $\Delta t$, they must also depend upon the thermal expansion characteristics of the material. Similarly if the resistivity ratios are functions of $\Delta t$, they must also depend upon the temperature-resistivity characteristics of the material. Up to moderately large values of the temperature interval $\Delta t$ these characteristics can be sufficiently defined by the thermal expansivity $\dot{L}$ and 
the temperature coefficient of resistivity $\dot{\rho}$, disregarding the variations of $\dot{L}$ and of $\dot{\rho}$ with temperature. Equation (42) can, therefore, be written

$$
R=f(L, \rho, \dot{L}, \dot{\rho}, \Delta t)
$$

for all bodies having the same generalized shape.

Applying the dimensional method, equation (45) becomes

$$
R=\frac{\rho}{L} \phi(\dot{L} \Delta t, \dot{\rho} \Delta t)
$$

which immediately reduces to equation (43) if $\dot{L}$ and $\dot{\rho}$ are independent of temperature, since $\Delta t$ is an arbitrary (external) variable and, therefore, likewise independent of temperature. From equation (46), therefore, we obtain the same final result as before, equation (44), for the relative change of resistance $R$ per unit change of the temperature $t$, although the intermediate steps are different on account of the nonuniform temperature distribution.

\section{EXAMPLES SOLVED WITH THE AID OF ISOTHERMAL EXPERIMENTS}

\section{VENTURI AIR-SPEED INDICATOR}

Let $p$ denote the differential pressure (or suction) generated either by the Venturi-static or Venturi-Pitot instrument on an aircraft traveling at a speed $v$ through air of density $\rho$ and let $P$ denote the dimensionless performance characteristic $p / \rho v^{2}$.

While the instrument dials are graduated for various air densities on the assumption that $P$ is a constant, it has been found that more accurate results can be obtained by treating $P$ as a function of viscosity $(12,13)$.

To investigate the effect of temperature on the performance characteristic $P$ for tubes of any given geometrical shape, we can begin by writing

$$
P=f(L, \mu, \rho, v)
$$

in which $L$ denotes a linear dimension, as, for example, the throat diameter, and $\mu$ denotes the viscosity of the air.

From equation (47) by dimensions

$$
P=\phi\left(\frac{L v \rho}{\mu}\right)
$$

Differentiating and following the same procedure as was employed in deriving equation (34) from equation (31), we obtain

$$
\dot{P}=K(\dot{L}+\dot{\rho}-\dot{\mu})
$$

where $K$ denotes the slope at any point of the graph obtained by plotting observed values of $\log P$ against $\log (L v \rho / \mu)$ at constant temperature.

Equation (49) will be recognized as an application of the graphical method, equation (34), taking $Q_{o}=P, \Pi_{o}=P, \Pi=L v \rho / \mu, Q=L, Q_{1}=\rho$, $Q_{2}=\mu, \alpha_{o}=0, \beta_{o}=0, \alpha=1$, and $\beta=-1$. 
The same result can be obtained in the following manner by the analytical method, equations (23) and (35). In equation (23) let $P=P, L=L, p_{1}=\rho, p_{2}=\mu$;

$$
\therefore \dot{P}=C_{0} \dot{L}+C_{1} \dot{\rho}+C_{2} \dot{\mu}
$$

in which

$$
\begin{aligned}
C_{o} & =\frac{\partial \log P}{\partial \log L} \\
C_{1} & =\frac{\partial \log P}{\partial \log \rho} \\
C_{2} & =\frac{\partial \log P}{\partial \log \mu}
\end{aligned}
$$

Obviously, a more convenient variable for experimenting than $L, \rho$, or $\mu$ will be the speed, $v$, since this is an external variable not involving any alteration of the body under test. We, therefore, seek to obtain, if possible, equivalent expressions for $C_{o}, C_{1}$, and $C_{2}$ in terms of the isothermal slope $\partial \log P / \partial \log v$, to replace the expressions given by equations (51) to (53).

In equation (35) let $Q_{o}=P, Q_{2}=v, \beta_{o}=0$, and $\beta=1$ throughout the following analysis, while for the purpose of evaluating $C_{o}$ we take $Q_{1}=L, \alpha_{o}=0, \alpha=1$; for evaluating $C_{1}$ take $Q_{1}=\rho, \alpha_{0}=0, \alpha=1$, and for evaluating $C_{2}$ take $Q_{1}=\mu, \alpha_{o}=0, \alpha=-1$.

Substituting these values successively in equation (35) gives

$$
\begin{aligned}
& \frac{\partial \log P}{\partial \log L}=\frac{\partial \log P}{\partial \log v}=C_{o} \\
& \frac{\partial \log P}{\partial \log \rho}=\frac{\partial \log P}{\partial \log v}=C_{1} \\
& \frac{\partial \log P}{\partial \log \mu}=-\frac{\partial \log P}{\partial \log v}=C_{2}
\end{aligned}
$$

Substituting in equation (50) the values of $C_{o}, C_{1}$, and $C_{2}$ given by equations (54) to (56)

$$
\dot{P}=\left(\frac{\partial \log P}{\partial \log v}\right)(\dot{L}+\dot{\rho}-\dot{\mu})
$$

This result confirms equation (49), since when $L, \rho$, and $\mu$ are held constant the slope $K$, representing $d \log P / d \log (L v \rho / \mu)$ reduces to $\partial \log P / \partial \log v$.

If we write $P \propto v^{n}$ as an approximation for a limited range of speeds, it follows that $K=n$. Now the exponent $n$ would be expected to lie between the limits of 0 for completely turbulent fluid motion (large throat diameter, high speed, and low altitude) and -1 for stream-line motion (small throat diameter, low speed, high altitude). For practical purposes $\dot{L}$ can be neglected in comparison with $\dot{\rho}$ and $\dot{\mu}$. Treating the atmosphere as an ideal gas of absolute temperature $\theta$, we find $\dot{\rho}=-1 / \theta$. Also as a fair approximation from $-40^{\circ}$ to $+40^{\circ}$ 
C., it has been shown (14) that $\dot{\mu}=+(4 / 5)(1 / \theta)$. Equations (49) and (57), therefore, reduce to the approximate form

$$
\dot{P}=-\frac{9}{5}\left(\frac{K}{\theta}\right)
$$

in which $K$ is a negative number (ranging from 0 to -1 ), so that $\dot{P}$ is always positive.

\section{SPRING STIFFNESS (COMPLEX STRESSES)}

When the shear modulus $\mu$ is added to the list of quantities already included in the stiffness problem, we obtain in place of equation (40) for all springs of the same generalized shape

$$
S=L E \phi\left(\frac{\mu}{E}\right)
$$

Differentiating leads to the result

$$
\dot{S}=\dot{L}+(1-K) \dot{E}+K \dot{\mu}
$$

in which $K$ is the slope of the graph obtained when the observed values of $\log (S / L E)$ are plotted against $\log (\mu / E)$ at constant temperature.

Equation (60) can also be derived by direct substitution in equation (34), taking $Q_{o}=S, \Pi_{o}=S / L E, \Pi=\mu / E, Q=\mu, Q_{1}=L, Q_{2}=E$, $\alpha_{o}=-1, \beta_{o}=-1, \alpha=0$, and $\beta=-1$.

An equivalent result in analytical form can be obtained by making the appropriate substitutions in equations (23) and (35).

If Poisson's ratio is used in place of $\mu / E$ in equation (59), we find $(1)^{9}$ with the aid of equations (12) and (13)

$$
\begin{aligned}
\dot{S} & =\dot{L}+C \dot{E}+C \dot{\sigma} \\
& =\dot{L}+\left[1+C\left(\frac{1+\sigma}{\sigma}\right)\right] \dot{E}-C\left(\frac{1+\sigma}{\sigma}\right) \dot{H}
\end{aligned}
$$

in which $C$ is the slope of the graph obtained by plotting log $(S / L E)$ against $\log \sigma$.

\section{VIBRATION FREQUENCY OF A LOADED SPRING (ELASTIC PENDULUM)}

A stiff spring having its natural frequency reduced by the addition of a localized mass $m$, has been used as a working standard for time intervals intermediate between the period of a tuning fork and that of a gravity pendulum.

If the stiffness of the spring can be measured at the effective point of application of the mass $m$, it can be shown by the method of dimensions that the frequency is given by

$$
n=\sqrt{\frac{S}{m}} \phi\left(\frac{m_{o}}{m}\right)
$$

${ }^{\text {}}$ See equation (7) of reference cited. 
in which $m_{o}$ denotes the mass of the spring itself. From equation (62) we readily find

$$
\dot{n}=\frac{1}{2} \dot{S}
$$

since evidently $m$ and $m_{o}$ are unaffected by temperature. Substituting from equation (60) into equation (63) gives

$$
\dot{n}=\frac{1}{2} \dot{L}+\frac{1}{2}\left(1-K_{s}\right) \dot{E}+\frac{1}{2} K_{s} \dot{\mu}
$$

in which $K_{s}$ is identical with $K$ of equation (60), the subscript being attached to call attention to the fact that the value is to be obtained from observations of stiffness rather than of frequency.

A more general solution can be obtained without reference to equation (62) as follows: Starting with a qualitative statement for systems of fixed generalized shape, we can write

$$
n=f(L, E, \mu, \rho, m)
$$

in which $\rho$ denotes the density of the spring material, the other quantities having the same interpretation as before. Then applying the method of dimensions to equation (65) we obtain

$$
n=\frac{1}{L} \sqrt{\frac{E}{\rho}} \phi\left(\frac{\mu}{E}, \frac{m}{\rho L^{3}}\right)
$$

It now appears immediately that the argument $m / \rho L^{3}$ is unaffected by temperature, since $m$ is essentially independent of temperature, while $\dot{\rho}=-3 \dot{L}$; differentiating, therefore, and disregarding $m / \rho L^{3}$, we find that

$$
\dot{n}=\frac{1}{2} \dot{L}+\frac{1}{2}(1-2 K) \dot{E}+K \dot{\mu}
$$

in which $K$ is the slope of the graph obtained by plotting $\log (n L \sqrt{\rho / E)}$ against $\log (\mu / E)$. This result agrees with equation (64) when $K_{s}$ is put equal to $2 K$, a relationship which can be deduced from the definitions of $K_{s}$ and $K$ in conjunction with equation (62).

Equation (67) can also be obtained by direct substitution in equation (34) after extending the latter to include the term $\left(\gamma K-\gamma_{0}\right) \dot{Q}_{3}$ and then putting $Q_{o}=n, Q=\mu, Q_{1}=L, Q_{2}=E, Q_{3}=\rho, \alpha_{o}=1, \alpha=O$, $\beta_{0}=-1 / 2, \beta=-1, \gamma_{o}=1 / 2$, and $\gamma=0$, and an equivalent result can be obtained, as in the two preceding examples, by making the appropriate substitutions in equations (23) and (35).

Turning back to the telephone diaphragm problem, it is interesting to note that equation (13) is a special case of equation (67) for which $K$ has the particular value $-3 / 7$.

\section{COEFFICIENT OF FRICTION OF A LUBRICATED JOURNAL BEARING}

As an example of a system which does not conform to the usual condition of geometrical similarity, ${ }^{10}$ consider the energy dissipated

${ }^{10}$ Sometimes erroneously assumed to be a prerequisite for the application of the method of dimensions. 
in the oil film which occupies the clearance space of a well-lubricated journal bearing. In general the oil film is not of a uniform thickness circumferentially, this being due to the eccentricity of the journal, which in turn depends on the viscosity of the oil, $\mu$, the speed of rotation, $n$, and upon the total load, $W$. There may also be a varying amount of cavitation at the open ends of the bearing; that is, the oil may not completely fill the clearance space between the journal and the bearing, even when liberally supplied. Nevertheless, under these conditions a simple relation has been found by the method of dimensions (15) which can be written

$$
f_{o}=\phi\left(\frac{\mu n L^{2}}{W}\right)
$$

In equation (68) $f_{o}$ is the coefficient of friction and $L$ is the length of the bearing, the subscript having been attached to represent the particular case in which the materials of both journal and bearing have the same thermal expansivity.

Differentiating, we obtain as was first shown by equation (22)

$$
\dot{f}_{o}=\left(\frac{\partial \log f_{o}}{\partial \log L}\right) \dot{L}+\left(\frac{\partial \log f_{o}}{\partial \log \mu}\right) \dot{\mu}
$$

From equation (68) either by inspection of by substituting in equation (35), selecting $n$ as the most convenient variable for experimental control,

$$
\begin{gathered}
\frac{\partial \log f_{o}}{\partial \log L}=2 \frac{\partial \log f_{o}}{\partial \log n} \\
\frac{\partial \log f_{o}}{\partial \log \mu}=\frac{\partial \log f_{o}}{\partial \log n} \\
\therefore \dot{f}_{o}=\left(\frac{\partial \log f_{o}}{\partial \log n}\right)(2 \dot{L}+\dot{\mu})
\end{gathered}
$$

or very approximately on account of the linear expansivity of the metal being small compared to the temperature coefficient of viscosity of the oil,

$$
\dot{f}_{o}=\left(\frac{\partial \log f_{0}}{\partial \log n}\right) \dot{\mu}
$$

Proceeding to the case of dissimilar metals where a still further departure from geometrical similarity occurs, we may consider first the effect of differential expansion on the clearance, $C$ (difference between bearing diameter and journal diameter). If $\Delta \dot{L}$ denotes the difference between the thermal expansivity of the bearing metal and that of the journal, while the diameter of the journal is represented by $D$, we have

$$
C \equiv\left(\frac{D}{C}\right) \Delta \dot{L}
$$

according as the bearing metal is free to expand without opposition, or restricted in some degree by stresses that are set up. 
While the connection between coefficient of friction and clearance can not be obtained from dimensional analysis, the following relation expressing the shearing resistance of a uniform film may be applied as a reasonable approximation for the purpose of computing the effect of small changes in temperature, viz:

$$
f \propto \frac{1}{C}
$$

Differentiating equation (75) we obtain for $\Delta \dot{f}$, the increment which must be added to equation (73) to take account of the change in clearance due to a rise of temperature,

$$
\Delta \dot{f}=-\dot{C}
$$

Referring to equation (74) and taking the equality sign as representing the maximum effect, substituting in equation (76) and adding the result to equation (73) gives finally

$$
\dot{f}=\left(\frac{\partial \log f}{\partial \log n}\right) \dot{\mu}-\left(\frac{D}{C}\right) \Delta \dot{L}
$$

Both terms on the right of equation (77) are normally negative for an oil-lubricated bearing operating on the stable side of the minimum coefficient of friction value, since $\dot{\mu}$ is negative and $\Delta \dot{L}$ positive. For an air-lubricated bearing $\dot{\mu}$ is positive.

The first term on the right of equation (77) has been readily obtained by the same methods demonstrated in the three examples immediately preceding. The second term will serve as a particular illustration of the general statement made in the paragraph following equation (23) regarding effects due to change of shape which can becalculated by conventional methods and then superposed on the solution obtained for a system of constant shape.

\section{EXTENSION TO DETERIMINE THE EFFECT OF PHYSICAL CONDITIONS OTHER THAN TEMPERATURE}

The variable quantity $t$ which has thus far been taken to represent the temperature is in fact entirely arbitrary and can equally well be interpreted as hydrostatic pressure, electrostatic, or magnetic field intensity, or any other measurable condition on which the properties of the system may depend. Temperature, however, can never (on the absolute scale) be made equal to zero, and, in general, for practical reasons, whatever other factor is investigated in any particular problem the temperature factor must be considered in addition.

As an example of the effect of pressure equation (44) may be written

$$
\dot{R}_{p}=\dot{\rho}_{p}-\dot{L}_{p}
$$

in which $\dot{R}_{p}$ denotes the relative increase in the electrical resistance of a body per unit increase of hydrostatic pressure; $\dot{\rho}_{p}$ the pressure 
coefficient of resistivity of the material and $\left(-\dot{L}_{p}\right)$ its linear compressibility. Replacing $\left(-\dot{L}_{p}\right)$ by its equivalent $(+k / 3)$, where $k$ is the ordinary volumetric compressibility, equation (78) becomes

$$
\dot{R}_{p}=\dot{\rho}_{p}+\frac{k}{3}
$$

Rewriting equation (44) with subscript $t$ indicating temperature effects,

$$
R_{t}=\dot{\rho}_{t}-\dot{L}
$$

From equations (79) and (80) we obtain for the change of resistance due to a simultaneous temperature rise $\Delta t$ and pressure increase $\Delta p$

$$
\frac{\Delta R}{R}=\left(\dot{\rho}_{t}-\dot{L}\right) \Delta t+\left(\dot{\rho}_{p}+\frac{k}{3}\right) \Delta p
$$

Equation (81) also enables us to compute either one of the coefficients $\dot{\rho}_{t}$ or $\dot{\rho}_{p}$ from a knowledge of the other when the expansivity and compressibility of the material are given and the actual change of resistance is observed.

\section{FURTHER QUEESTIONS FOR INVESTIGATION}

A logical continuation of the present study might proceed along somewhat the following lines:

(a) Further practical applications.

(b) Compilation of tables of data for the requisite thermal coefficients, such as $\dot{L}, \dot{E}, \dot{\mu}$, etc.

(c) Formulation of precedure for computing $\dot{r}_{1} \cdots \dot{r}_{l}$ for systems of variable generalized shape.

(d) Extension to bodies of nonuniform temperature.

(e) Extension to include the effect of large changes in temperature.

(f) Extension to other physical observations besides properties of bodies.

\section{REFERENCES}

1. Hersey, M. D., The Theory of the Stiffness of Elastic Systems, J. Wash. Acad. Sci. 6, pp. 569-575; 1916.

2. Hersey, M. D., Note on the Vibration Frequencies of Elastic Systems, J. Wash. Acad. Sci. \%, pp. 437-445; 1917.

3. Rayleigh, Theory of Sound (2d ed.), London, MacMillan, 1, 221 (a); 1894.

4. Buckingham, E., Physically Similar Systems, J. Wash. Acad. Sci. 4, pp. 347-353; 1914; Phys. Rev. 4, pp. 345-376; 1914.

5. Buckingham, E., Model Experiments and the Forms of Physical Equations, Trans. Am. Soc. Mech. Eng. 3\%, pp. 263-296; 1915.

6. Buckingham, E., Notes on the Method of Dimensions, Phil. Mag. 42, pp. 696-719; 1921.

7. Hersey, M. D., A Relation Connecting the Derivatives of Physical Quantities, J. Wash. Acad. Sci. 6, pp. 620-629; 1916; B. S. Sci. Papers 15, (S331) pp. 21-29; 1919.

8. Rayleigh, The Principle of Similitude, Nature 95, pp. 66-68; 1915.

9. Bridgman, P. W., Dimensional Analysis, New Haven, Yale University Press; 1922 .

10. Hersey, M. D., Note on a General Method for Determining Properties of Matter, J. Wash. Acad. Sci. 12, pp. 167-172; 1922. 
11. Buckingham, E., Dimensional Analysis, Phil. Mag. 48, pp. 141-145; 1924.

12. Hersey, M. D., Hunt, F. L., and Eaton, H. N., The Altitude Effect on AirSpeed Indicators, Report No. 110, Nat. Adv. Com. for Aero., Sixth Annual Rept., pp. 691-717; 1920.

13. Hersey, M. D., Theory of Air-Speed Indicators, Prem. Cong. Navig. Aérienne, Paris; 1921; Rapport II, pp. 79-86.

14. Hersey, M. D., Variation of Fluid Properties in Aerodynamics, Internat. Air Cong., London; 1923; Report, pp. 414-421.

15. Hersey, M. D., Laws of Lubrication of Horizontal Journal Bearings, J. Wash. Acad. Sci. 4, pp. 542-552; 1914.

Washington, February 26, 1929. 